\title{
Examination of the Importance of Pro-453 in Human Dihydrolipoamide Dehydrogenase Predicted from the Three-Dimensional Structure
}

\author{
Hakjung Kim \\ Department of Chemistry, College of Natural Science, Daegu University, Kyoungsan 712-714, Korea \\ "E-mail:hjkim@daegu.ac.kr \\ Received February 23, 2006
}

Key Words : Dihydrolipoamide dehydrogenase, Pyridine nucleotide-disulfide oxidoreductase, $\alpha$-Keto acid dehydrogenase complex, Site-directed mutagenesis

Dihydrolipoamide dehydrogenase (E3) (dihydrolipoamide: $\mathrm{NAD}^{+}$oxidoreductase; EC 1.8.1.4), along with glutathione reductase and trypanothione reductase, belongs to a group of flavoenzymes known as the pyridine nucleotide-disulfide oxidoreductase family. ${ }^{1}$ These enzymes are homodimeric proteins containing an active disulfide center and a FAD in each of their subunits. Each active site of these enzyme dimers contains residues from both subunits. ${ }^{2-4}$ One subunit contributes most of the active site residues, including the active disulfide center and the FAD and $\mathrm{NAD}^{+}$(NADH) binding sites, while the other subunit provides a proton acceptor/donor, His residue, and its hydrogen bonding partner, Glu residue.

His-452 in human E3 acts as a proton acceptor/donor and Glu-457 stabilizes His-452 by acting as a hydrogen bonding partner. $^{2}$ The next residue of His-452 is Pro-453. The Pro residue is absolutely conserved in E3s and other pyridine nucleotide-disulfide oxidoreductases, along with the His and Glu residues. The recently reported three-dimensional structure of the human E3 provides more detailed information about Pro- $453 .^{5}$ The structure shows that the peptide bond between His-452 and Pro-453 is in a cis configuration and the N3 atom of FAD forms a hydrogen bond with the oxygen atom of the cis peptide bond. It has been predicted that a mutation at Pro-453 could lead to a change in the cis configuration of the peptide bond that would affect the structure and function of human E3.

To examine the importance of Pro-453 in the structure and function of human E3, predicted from its three-dimensional structure, Pro-453 was mutated to Val by site-directed mutagenesis. Val was selected since it has a similar size side chain to that of Pro. Two polymerase chain reactions (PCR) were carried out using the primer pairs $A$ with $B$ and $C$ with D, respectively. Primer A (5'-TCCCAACGACCGAAAACCTG-3') and Primer D (5'-GCCAAAACAAGCCAAGCTTGG-3') are sense and anti-sense oligomers, respectively, which can be used to amplify the whole E3 gene sequence.

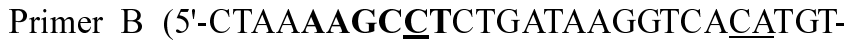
GCATG-3': the mismatched bases are underlined and the mutated HindIII site is written in bold type) is an anti-sense oligomer with mutation to convert Pro-453 to Val. Primer C (5'-CATGCACATGTGACCTTATCAGAGGCTTTTAG-3': the mismatched bases are underlined and the mutated
HindIII site is written in bold type) is the corresponding sense oligomer of Primer B. The reaction was carried out in a programmable PCR machine using the human E3 expression vector as a template as described elsewhere. ${ }^{6}$ The PCR generated fragment (1501 bp) containing the E3 sequence with the mutation was digested with EcoRI and BamHI and ligated with the expression vector lacking the normal EcoRI/ BamHI sequence. This ligation resulted in the construction of the Val-453 mutant expression vector. Mutation was confirmed by DNA sequencing. The expression and purification of the mutant were performed as described elsewhere. ${ }^{7}$ Purification steps were followed by SDS-polyacrylamide gel electrophoresis (data not shown). The gel revealed that the mutant was highly purified.

The E3 activity of the mutant was determined as 0.37 unit/ $\mathrm{mg}$ at the saturated substrate concentrations of $2 \mathrm{mM}$ dihydrolipoamide and $3 \mathrm{mM} \mathrm{NAD}$ at $37{ }^{\circ} \mathrm{C}$ in a $50 \mathrm{mM}$ potassium phosphate buffer ( $\mathrm{pH} 8.0$ ) containing $1.5 \mathrm{mM}$ EDTA. This value was about 1,650-fold lower than that of normal human E3 activity determined under the same conditions. Even though it was difficult to determine the steady-state kinetic parameters, due to the very low activity and instability of the mutant, the 1,650-fold decrease in E3 activity of the mutant clearly indicated that the Pro-453 is very important to the human E3 catalytic function.

To examine any structural changes occurring in the mutant, UV-visible absorption and fluorescence spectroscopies were performed. Due to the prosthetic group FAD, human E3 has a characteristic UV-visible absorption spectrum of flavoproteins as shown in Figure 1. The overall shape of the mutant spectrum (solid line) was very similar to that of the wild-type human E3 spectrum (dashed line). However, a noticeable alteration was found in the region of the unique shoulder, as indicated by the arrow in Figure 1. The unique shoulder, clearly observed in the wild-type human E3 spectrum from $465 \mathrm{~nm}$ to $485 \mathrm{~nm}$, was largely diminished in the mutant spectrum. It seems that the spectrum of the mutant became similar to that of FAD (dotted line) even though the spectrum was still more similar to the wild-type human E3 spectrum. This alteration in the UVvisible spectrum of the mutant implied that structural changes occurred in the FAD binding region of the mutant. The mutation of Pro-453 to Val probably affected the cis 


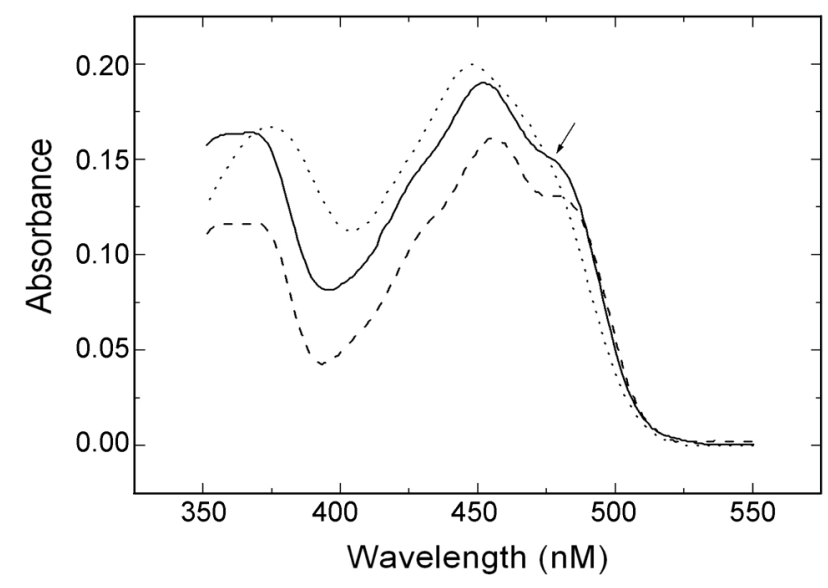

Figure 1. UV-visible spectra of the Val-453 mutant (16.3 $\mu \mathrm{M}$, solid line) and wild-type (14.2 $\mu \mathrm{M}$, dashed line) recombinant human E3s and FAD (17.7 $\mu \mathrm{M}$, dotted line). The spectra were recorded using Shimazu UV 160A spectrophotometer and the data from $350 \mathrm{~nm}$ to $550 \mathrm{~nm}$ were transferred to an ASCII file and the spectra were drawn using the MicroCal Origin program.

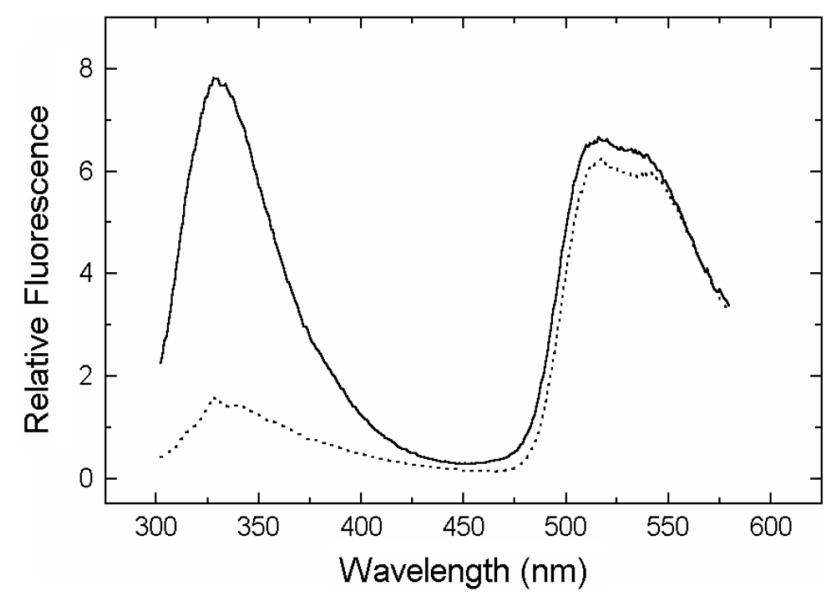

Figure 2. Fluorescence spectra of the Val-453 mutant $(22.6 \mu \mathrm{M}$, solid line $)$ and wild-type $(12.5 \mu \mathrm{M}$, dashed line $)$ recombinant human E3s. Enzymes were excited at $296 \mathrm{~nm}$ and the emissions were observed from $302 \mathrm{~nm}$ to $580 \mathrm{~nm}$. The data were transferred to an ASCII file and the spectra were drawn using the MicroCal Origin program.

configuration of the peptide bond. The resulting structural changes interfered with the interaction between FAD and the cis peptide bond.

More evidence of the structural changes in the mutant came from the fluorescence study. When enzymes were excited at $296 \mathrm{~nm}$, two fluorescence emissions were observed for both mutant and wild-type E3s, as shown in Figure 2. The first emission from $302 \mathrm{~nm}$ to $400 \mathrm{~nm}$ was mainly due to Trp. The second emission from $480 \mathrm{~nm}$ to over $550 \mathrm{~nm}$ was due to FAD. When the fluorescence spectra of E3s were compared, a noticeable difference was found in the ratio between relative intensities of the first and second fluorescence emissions. The ratio (about 0.8 ) between relative intensities of the first and second fluorescence emissions of the mutant (solid line) was much lower than that (about 4) of the wild-type enzyme (dashed line). This difference in the fluorescence spectrum patterns between the mutant and wild-type enzymes implied again that structural changes occurred in the mutant. The structural changes, which led to the previously altered UV-visible spectrum of the mutant, could also have caused these alterations in the fluorescence spectrum of the mutant. The degree of relative intensity increase of the first emission of the mutant was higher than that of the relative intensity decrease of the second emission. There are two Trp residues (Trp-197 and Trp-366) in human E3. Trp-197 is located in the NAD domain, while Trp-366 is located near the boundary between the central and interface domains. $^{2}$ The structural changes due to the mutation of Pro453 to Val could have affected the overall structure of human E3 so that the first florescence emission of these two Trp residues was largely altered. Exact structural changes occurring due to the mutation could be revealed by an X-ray crystallographic study.

Here, the importance of Pro-453 in the structure and function of human E3, predicted from the three-dimensional structure, has been examined using site-directed mutagenesis, E3 activity measurement and spectroscopic methods. The following conclusions can be drawn. 1. The conservation of Pro-453 in human E3 is very important to the catalytic function of the enzyme. 2. The predicted structural changes in the FAD binding region of human E3 due to the mutation at Pro-453 were supported by the altered UV-visible spectrum of the mutant. 3 . The mutation at Pro-453 affected the overall structure of human E3. These conclusions can be applied to other pyridine nucleotide-disulfide oxidoreductases with a similar structure and catalytic mechanism of human E3, such as glutathione reductase and trypanothione reductase, since the Pro residues are absolutely conserved in these enzymes and they have similar three-dimensional structures.

Acknowledgements. The author thanks Dr. Mulchand S. Patel (State University of New York, Buffalo) for a generous gift of an E. coli XL1-Blue containing a human E3 expression vector. This research was supported in part by the Daegu University Research Grant, 2005.

\section{References}

1. Williams, C. H., Jr. Enzymes, 3rd ed; Boyer, P., Ed.; Academic Press: $1976 ;$ p 89.

2. Jentoft, J. E.; Shoham, M.; Hurst, D.; Patel, M. S. Proteins: Struct. Funct. Genet. 1992, 14, 88.

3. Thieme, R.; Pai, E. F.; Schirmer, R. H.; Schulz, G. E. J. Mol. Biol. 1981, 152, 763 .

4. Schierbeek, A. J.; Swarte, M. B. A.; Dijkstra, B. W.; Vriend, G.; Reed, R. J.; Hol, W. G. J.; Drenth, J. J. Mol. Biol. 1989, 206, 365.

5. Brautigam, C. A.; Chuang, J. L.; Tomchick, D. R.; Machius, M.; Chuang, D. T. J. Mol. Biol. 2005, 350, 543.

6. Kim, H. J. Biochem. Mol. Biol. 2002, 35, 437.

7. Kim, H. J. Biochem. Mol. Biol. 2005, 38, 248. 